\title{
MICROMACHINED MEMBRANE PARTICLE FILTERS
}

\author{
X. Yang, J. M. Yang*, X. Q. Wang, E. Meng, Y. C. Tai, and C. M. Ho* \\ Caltech Micromachining Laboratory \\ Electrical Engineering, 136-93 \\ California Institute of Technology \\ Pasadena, CA 91125 , USA \\ *Department of Mechanical and Aerospace Engineering \\ University of California, Los Angeles \\ Los Angeles, CA 90095, USA
}

\begin{abstract}
We report here several particle membrane filters $\left(8 \times 8 \mathrm{~mm}^{2}\right)$ with circular, hexagonal and rectangular through holes. By varying hole dimensions from 6 to $12 \mu \mathrm{m}$, opening factors from 4 to $45 \%$ are achieved. In order to improve the filter robustness, a composite silicon nitride/Parylene membrane technology is developed. More importantly, fluid dynamic performance of the filters is also studied by both experiments and numerical simulations. It is found that the gaseous flow through the filters depends strongly on opening factors, and the measured pressure drops are much lower than that from numerical simulation using the Navier-Stokes equation. Interestingly, surface velocity slip can only account for a minor part of the discrepancy. This suggests that a very interesting topic for micro fluid mechanics research is identified.
\end{abstract}

\section{INTRODUCTION}

Filtration and collection of particles is an important process in airborne particle sampling. This work focuses on airborne particles in the range of 1 to $10 \mu \mathrm{m}[1]$, and micromachined membranes with perforations are ideal candidates for such filters. Although several MEMS filters $[2,3,4]$ have been reported in the past, a comprehensive study of their strength and fluid dynamic performance is not available. For MEMS membrane filters to be effective, various requirements must be met. They must be mechanically robust so as to stand a potentially high pressure drop. The filter opening factor must be high to allow for a large amount of air flow. Finally, the pressure drop, and hence the power loss, has to be low. As a result, factors including hole dimension, shape, membrane thickness and the opening area factor ( $\beta=$ area of holes/total area) decide the main performance of the filters. Large holes and a large opening factor will decrease the pressure drop and increase the flow rate, but decrease the strength of the membrane. Different hole shapes will not only change the flow rate and pressure drop, but also the stress concentration level in the membrane, and thus the strength of the filter as well. More interestingly, our work has also identified the importance of micro fluid dynamics factors that micro gas flow in small holes, although not yet fully understood, should also be considered. The goal of this project is to develop new structures and fabrication processes to enhance the strength of the filters and to establish a guideline to design an optimal membrane filter by studying the fluid dynamic performance experimentally and numerically.

\section{FABRICATION}

The filters are fabricated by using the process shown in Fig. 1. First, a layer of $1 \mu \mathrm{m}$ thick LPCVD silicon

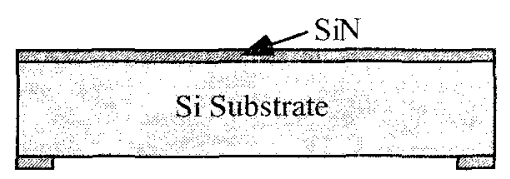

(a) SiN Deposition

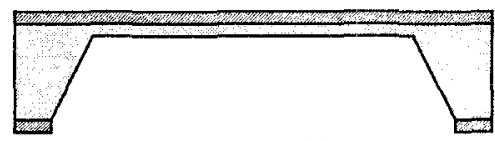

(b) $\mathrm{KOH}$ Etching

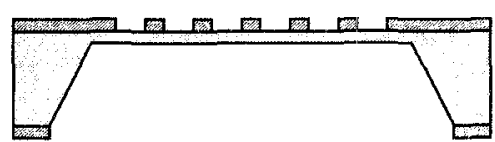

(c) SiN Patterning

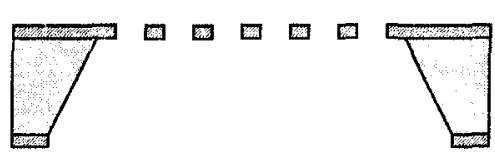

(d) Si Etching

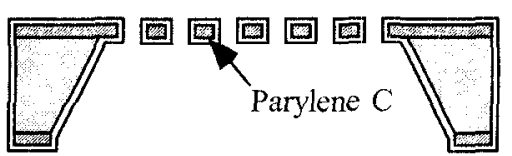

(e) Parylene C Deposition

Fig. 1 Fabrication Process 
Table 1 Filter Design

\begin{tabular}{|c|c|c|c|c|c|}
\hline Filter & $\begin{array}{l}\text { Top View }(8 \mathrm{~mm} \times 8 \mathrm{~mm}) \\
\text { : Non-Filtering Area } \\
\square \text { : Filtering Area }\end{array}$ & $\begin{array}{l}\text { Cross-sectional View } \\
\text { : Silicon Nitride } \\
\text { 圆: Parylene Coating }\end{array}$ & $\begin{array}{l}\text { Hole Size } \\
d(\mu \mathrm{m}) \text { or } \\
\mathrm{a} \times \mathrm{b}\left(\mu \mathrm{m}^{2}\right)\end{array}$ & $\begin{array}{c}\text { Opening Factor } \\
\beta(\%)\end{array}$ & $\begin{array}{l}\text { Hole } \\
\text { Shape }\end{array}$ \\
\hline 1 & $5.3 \mathrm{~mm}$ & $(2 \mu \mathrm{m}+2 \times 1 \mu \mathrm{m})$ & 6 & 4 & Circular \\
\hline 2 & $\pi^{2.3 \mathrm{~mm}}$ & $\stackrel{(2 \mu \mathrm{m})}{\mathrm{m}}$ & $8 \sim 8.8$ & 8 & Circular \\
\hline 3 & 原 $5.3 \mathrm{~mm}$ & $\stackrel{(2 \mu \mathrm{m})}{\square}$ & 10.6 & 13 & Circular \\
\hline 4 & & $=$ & 8 & 20 & Hexagonal \\
\hline 5 & & $\quad(1 \mu \mathrm{m})$ & 12 & 45 & Hexagonal \\
\hline 6 & & $-(1 \mu \mathrm{m})$ & $5 \times 20$ & 39 & Rectangular \\
\hline 7 & & $(1.1 \mu \mathrm{m}+2 \times 1 \mu \mathrm{m})$ & $3 \times 18$ & 21 & Rectangular \\
\hline
\end{tabular}

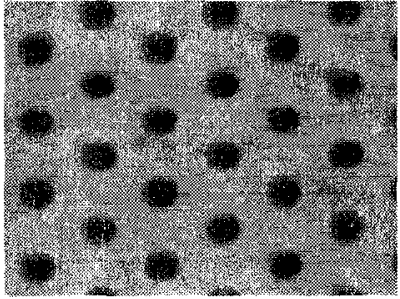

(a) Circular

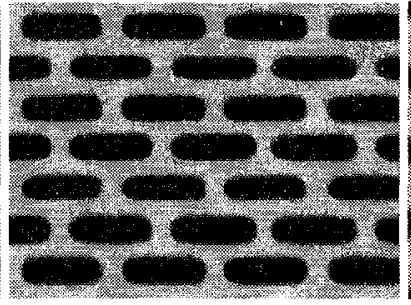

(b) Rectangular

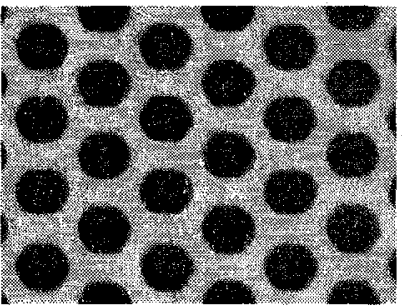

(c) Hexagonal

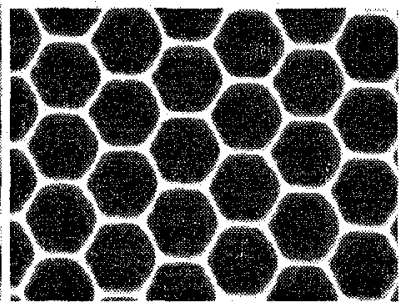

(d) Hexagonal

Fig. 2 Photographs of the Fabricated Filters

nitride ( $\mathrm{SiN})$ is deposited at $850{ }^{\circ} \mathrm{C}$ with a $\mathrm{SiH}_{2} \mathrm{Cl}_{2}: \mathrm{NH}_{3}$ gas flow ratio of $4: 1$ to achieve low stress [5]. Windows on the backside of the wafers are opened using plasma etching. The wafers are then etched in potassium hydroxide $(\mathrm{KOH})$ until only $20 \mu \mathrm{m}$ silicon is left. Next, an array of filtering holes is etched into the silicon nitride layer on the front side of the wafer using reactive ion etching (RIE). Then the wafers are diced and etched in $\mathrm{KOH}$ again to remove the remaining silicon and free the membrane. By dicing the wafers before the membranes are freed, the possibility of damaging the fragile membranes during the dicing is avoided. Finally, a layer of poly-p-xylylene (Parylene $C$, Specialty Coatings Systems) polymer is deposited conformally over the wafers or individual dies. This layer of Parylene serves two purposes. First, it can greatly improve the strength of the membrane filters. Second, since the Parylene deposition is conformal, different hole sizes can be obtained from the same base filter by varying the thickness of the Parylene layer.

\section{DESIGN}

Using the fabrication process described above, several membrane filters as large as $8 \times 8 \mathrm{~mm}^{2}$ have been made. The membrane filters are perforated with holes of varying shapes such as circles, rectangles and hexagons. As shown in Table 1, the opening factor of the filters is in the range of 4 to $45 \%$ with different hole sizes and pitches. As shown later in the testing section, the pressure drop and the power requirement of the filters to sustain a desired flow rate strongly depend on the opening factor. A high opening factor is necessary to achieve low pressure drop and high flow rate in the filters, and the opening factor increases as the hole size increases. However, the maximum hole size is determined by the targeted particle size. Thus there is a tradeoff between opening factor and hole size. Typical filter parameters are, for example, $10.6 \mu \mathrm{m}$ diameter circular holes with an opening factor of $13 \%$. Hexagonal holes offer a higher opening factor than circular holes, but their geometry results in greater 


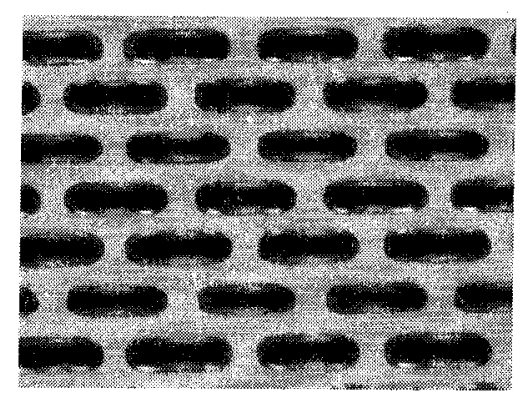

Fig. 3 Photograph of a Parylene Coated Filter 6 in Fig. 2 (b)

Table 2 Filter Strength (Filter $6, \beta=39 \%$ )

\begin{tabular}{|c|c|}
\hline $\begin{array}{c}\text { Parylene } \\
\text { Thickess } \\
(\mu \mathrm{m})\end{array}$ & $\begin{array}{c}\text { Burst Pressure } \\
(\mathrm{PSI})\end{array}$ \\
\hline 0 & 0.9 \\
\hline 1.38 & 1.9 \\
\hline 2.69 & 4.2 \\
\hline
\end{tabular}

stress concentration in the membrane, which decreases the strength of the filters. Rectangular holes can give a large range of opening factors without changing the filtering threshold as long as one dimension of the rectangular holes is kept constant. But they suffer from the same stress concentration problem as hexagonal holes. Fig. 2 shows photographs of the fabricated filters with (a) circular (b) rectangular and (c, d) hexagonal holes.

\section{STRENGTH}

Since the filters will be used for particle collection, they have to be strong enough to stand the aerodynamic load. The strength of the filters is studied and compared to that of flat silicon nitride membranes with identical dimensions but without perforations.
High pressure air flow is passed through the filters. By increasing the flow until the filter breaks, the "burst pressure" is then determined as the differential pressure at burst. For example, a $8 \mathrm{~mm} \times 8 \mathrm{~mm} \times 1 \mu \mathrm{m}$ membrane bursts at 5.5 psi while Filter 6 (Fig. 2 (b)) in Table 1 bursts at 0.9 psi. Filter 6 has a lower burst pressure due to the stress concentration in the membrane caused by the perforations. To improve the burst pressure, a layer of Parylene is conformally deposited on the filters. Fig. 3 is a picture of a Filter 6 coated with $1.38 \mu \mathrm{m}$ of Parylene. The shadows around every hole clearly show the conformal nature of the Parylene deposition. Table 2 shows that the thicker the membrane, the higher the burst pressure. With $2.69 \mu \mathrm{m}$ thick Parylene deposition, the burst pressure of Filter 6 is increased to 4.2 psi which is more than four times that of an uncoated one and is comparable to that of an unperforated membrane.

\section{FLUID DYNAMIC TESTING}

To test the fluid dynamic performance, a small wind tunnel shown in Fig. 4 is designed and built. The wind tunnel consists of inlet, settling chamber, contraction, testing chamber, diffuser and suction fan. A prefilter is inserted at the inlet of the wind tunnel to prevent the unwanted particles in the air from clogging the filter under test. The contraction section is carefully designed so that a uniform mean flow in the test section can be achieved and there is no separation of the flow at the inlet of the test section. Several different commercially available suction fans (computer fan, hair dryer fan, etc.) are used to provide flow up to 1001 liter $/ \mathrm{min} / \mathrm{cm}^{2}$. The test section has a length of $30 \mathrm{~cm}$ and a crosssection of $8 \mathrm{~mm}$ by $8 \mathrm{~mm}$. The filter is tested at the point $15 \mathrm{~cm}$ downstream from the contraction. Pressure drop across the filters is measured by using a manometer which has a resolution of $0.005 \mathrm{mmHg}$. The flow rate is measured by the Venturi tube principle. By measuring static pressure before $\left(\mathrm{P}_{1}\right)$ and after the contraction $\left(\mathrm{P}_{2}\right)$ of the wind tunnel using a MKS Baratron differential pressure transducer and solving the continuity equation,

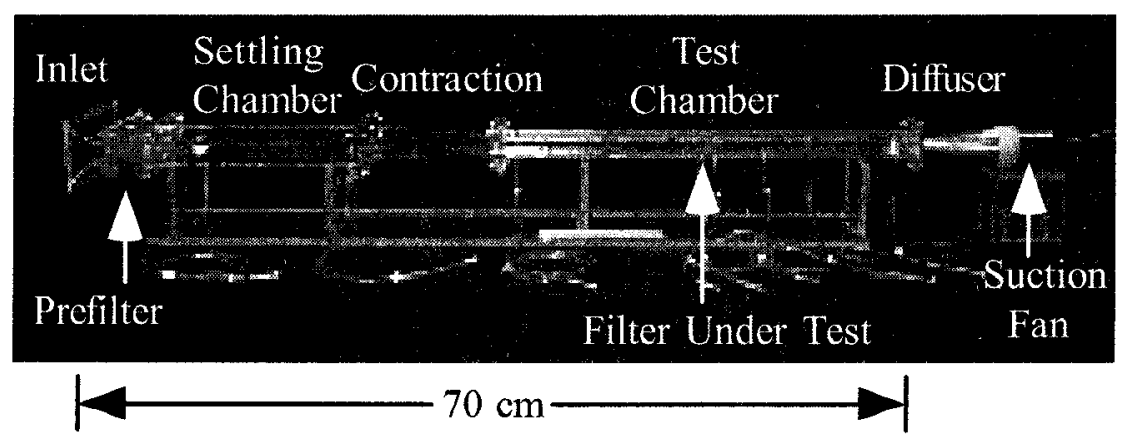

Fig. 4 Wind Tunnel Testing Setup 


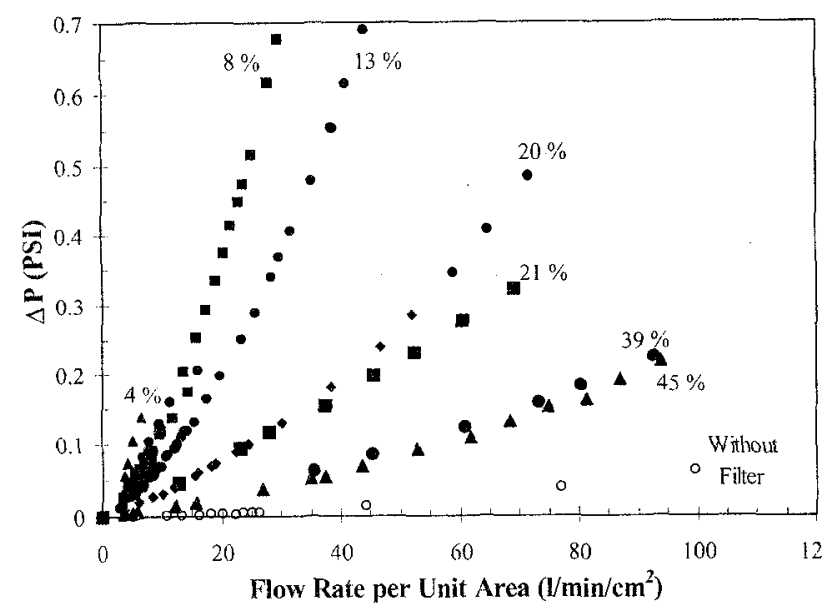

Fig. 5 Pressure Drop $V s$. Flow Rate per Unit Area of Filters with Different Opening Factors

$U_{1} A_{1}=U_{2} A_{2}$

and the Bernoulli equation,

$P_{1}+\frac{\rho U_{1}^{2}}{2}=P_{2}+\frac{\rho U_{2}^{2}}{2}$,

where $U$ is the average velocity across the cross-section, $A$ is the area of the cross-section, $\rho$ is the density of air, and $P$ is the pressure, the volumetric flow rate $Q$ can then be calculated as,

$$
Q=U_{2} A_{2}=A_{2} \sqrt{\frac{2\left(P_{1}-P_{2}\right)}{\rho\left(1-A_{2}^{2} / A_{1}^{2}\right)}} .
$$

Pressure drop as a function of the flow rate per unit area for seven different filters is plotted in Fig. 5. The power required to sustain a desired flow rate is calculated by multiplying the pressure drop by the volumetric flow rate and the result is shown in Fig. 6. As expected, it can be seen that the pressure drop and the power requirements strongly depend upon the opening factor of the filter.

\section{NUMERICAL CALCULATION}

One of the goals of this project is to establish a design guideline for membrane filters. As seen from filter testing results, the pressure drop and the power requirement depend upon the dimension $(d)$ and shape of the holes, the thickness of membrane $(t)$ and the filter opening factors $(\beta)$. In non-dimensional analysis, this can be shown as,

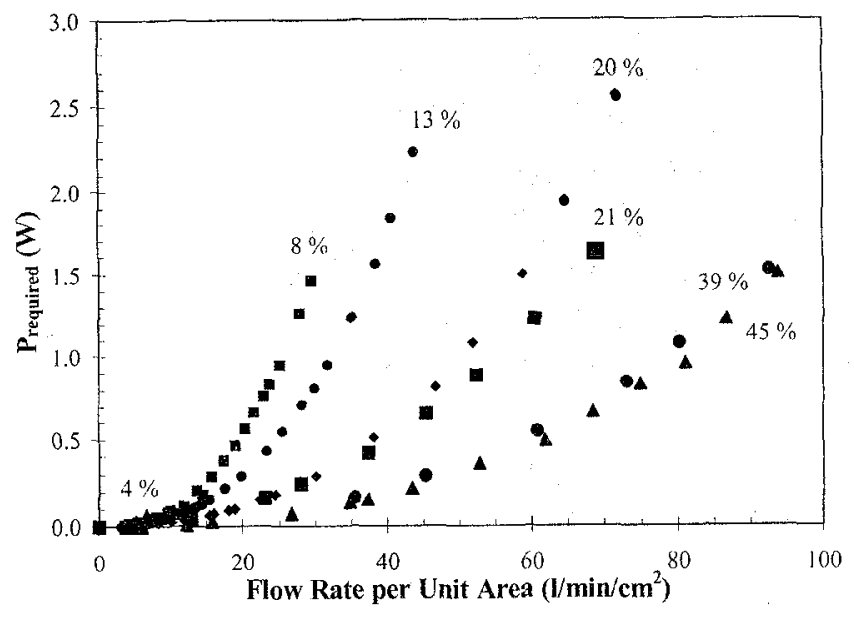

Fig. 6 Power Requirement $V_{S}$. Flow Rate per Unit Area of Filters with Different Opening Factors

$K=\frac{\Delta P}{\frac{1}{2} \rho U_{\text {in }}^{2}}=f(\operatorname{Re}, \beta, t / d)$,

where $K$ is the pressure coefficient, $\Delta P$ is the pressure drop, $\operatorname{Re}$ is the Reynolds number which is defined as,

$\operatorname{Re}=\frac{U_{h} d}{v}$,

where $U_{h}$ is the average velocity across the hole, $d$ is the diameter of the hole and $v$ is the kinematic viscosity of air. In our filter testing experiments, $\mathrm{Re}$ is in the range of 1 to 40 .

Wieghardt [6] and Schubauer et al. [7] proposed empirical formulas for $K$, but their formulas are only valid for large Reynolds number flow ( $R e>60$ ) and large holes $(d>100 \mu \mathrm{m})$. Recently, Hasegawa et al. [8] investigated the pressure drop through a single orifice as small as $8.8 \mu \mathrm{m}$. However, all the testing is conducted with liquid. Therefore, none of the existing empirical formulas can model the fluid dynamic behavior of our membrane filters. Numerical simulation is utilized to aid in determining the empirical formula for the micromachined membrane filters with small holes and operated in low Reynolds number flow regimes. Considering that the filter can be regarded as an array of small holes, we perform numerical calculations around a single axisymmetric cylindrical orifice by using the following model in CFDRC (Computational Fluid Dynamics Research Corporation) software.

By assuming a no-slip boundary condition at the surface of the filter, viscous laminar flow and varying the following parameters, 


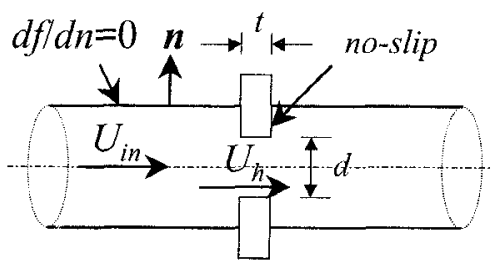

$10 \%<\beta<45 \%$

$0.08<t / d<0.5$

$1<\operatorname{Re}<100$

the pressure drop is calculated and plotted in Fig. 7. It is found that the results can be expressed as a single curve regardless of the geometrical parameters if the following non-dimensional form is used,

$$
K \beta^{2}\left(\frac{t}{d}\right)^{n}=f\left(\frac{U_{h} d}{v}\right) .
$$

As shown in Fig. 7, fitting the curve results in the following formula,

$$
K=\frac{\Delta P}{\frac{1}{2} \rho U_{i n}^{2}}=\beta^{-2}\left(\frac{t}{d}\right)^{0.28}\left[73.5 \frac{v}{U_{h} d}+1.7\right]
$$

Equation (7) shows that the pressure drop coefficient varies inversely as the second power of the opening factor $\beta$. Thus, it can be concluded that the opening factor is the most important parameter in determining the pressure drop. This is consistent with the results shown in Fig. 5.

\section{RESULTS AND DISCUSSIONS}

To examine the validity of the equation obtained from

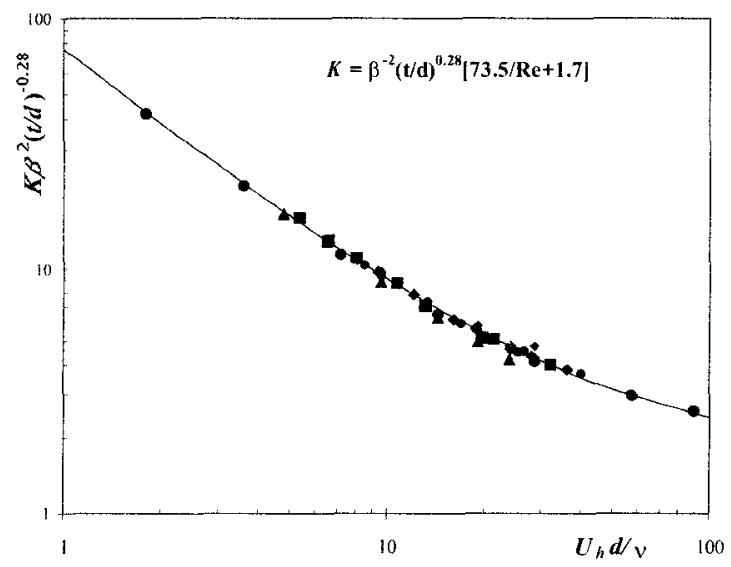

Fig. 7 Numerical Calculation for Various Geometric Factors

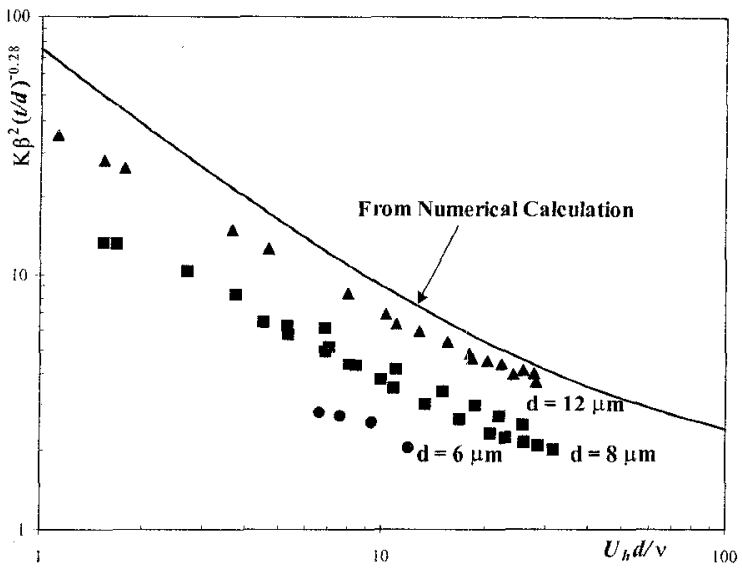

Fig. 8 Experiments VS. Numerical Calculations

simulations, it is necessary to compare the numerical calculations with experimental results. Plotted in Fig. 8 are pressure drop coefficients from the numerically simulated formula and experimental pressure drop coefficients of filters with $6 \mu \mathrm{m}, 8 \mu \mathrm{m}$, and $12 \mu \mathrm{m}$ holes. It can be seen that the deviation from the numerical result increases as the dimension of the holes decreases. This indicates that some of the assumptions used in the numerical calculation are not valid. Since the filter holes are very small, one possibility is that the no-slip boundary condition does not hold The conventional way to check the possibility of slip at the wall is to calculate the Knudsen number $(K n)$ of the flow field. Knudsen number is defined as the ratio of the mean free path $(\lambda)$ of working fluid (air, in this case, $\lambda=0.0685 \mu \mathrm{m}$ at $1 \mathrm{~atm}$ ) to the physical dimension of flow field (hole size, d). It is traditionally known that slip occurs at the wall only if the Knudsen number is larger than 0.01. The Knudsen number is plotted as a function of the hole size of the filters in Fig. 9. It is shown that Knudsen number of the filters ranges from 0.005 to 0.015 . The Knudsen number in the case of $d=$ $8 \mu \mathrm{m}$ is approximately 0.009 , which means that slip could be significant.

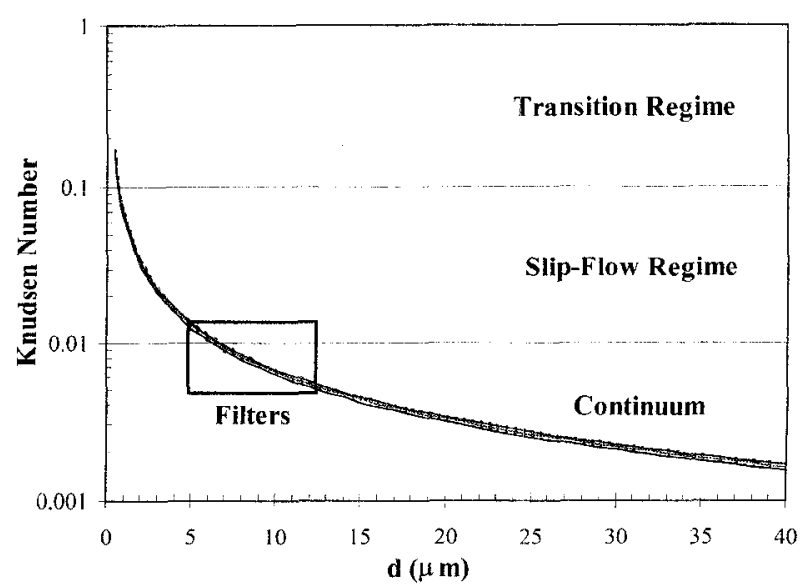

Fig. 9 Knudsen Number $V s$. Filter Hole Size 


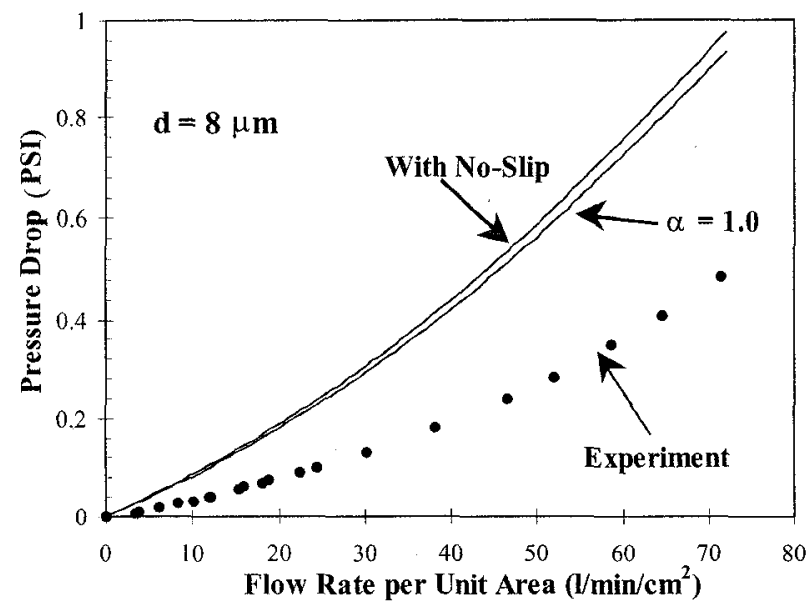

Fig. 10 Numerical Calculation Results with Slip Boundary Condition

To determine if the no-slip boundary condition is truly the cause of the large discrepancy between numerical calculations and experimental results, additional numerical calculations are performed that include the following slip boundary condition,

$u_{w}= \pm\left.\frac{2-\alpha}{\alpha} \lambda \frac{d u}{d y}\right|_{w}$

where $\alpha$ is the accommodation constant (which ranges from 0 to 1) and $\lambda$ is the mean free path of air. The result is shown in Fig. 10. Even though the slip boundary condition is included and $\alpha$ is varied between 0 and 1 , there is still a large difference. This suggests that some other mechanisms must be attributed to this effect. For example, in numerical calculation, no consideration is given to surface roughness and interaction between the wall surface molecules and the molecules in the air flow. Also, to simplify the simulation process, the holes and boundary between holes are assumed to be circular even though in reality the filter holes are hexagonal. So far, however, no specific mechanism has been identified that satisfactorily explains the discrepancy. More work is underway to determine which one of the above two mechanisms or both are responsible.

\section{CONCLUSION}

We have designed, fabricated and tested several particle membrane filters $\left(8 \times 8 \mathrm{~mm}^{2}\right)$ with circular, hexagonal and rectangular filtering holes. By varying hole dimensions from 6 to $12 \mu \mathrm{m}$, opening factors from $4 \%$ to $45 \%$ are achieved. A composite silicon nitride/Parylene membrane technology is developed to enhance filter strength. Fluid dynamic performance of the filters has been studied extensively through experiments and numerical simulations. It is found that the gaseous flow in the filters strongly depends on opening factors. Furthermore, the measured pressure drops are much lower in comparison to numerical simulation results. Numerical calculation with a slip boundary condition shows that surface velocity slip can only account for a minor part of the difference. Although the membrane filters and their functionality has been successfully developed, more work is needed to find out the fluid dynamic mechanism that causes this discrepancy.

\section{ACKNOWLEDGMENTS}

This work is supported by the DARPA MICROFLUMES program under Naval Ocean Systems Center Contract N66001-96-C-83632. The authors would like to thank Trevor Roper for help with processing.

\section{REFERENCES}

[1] C. N. Davies, "Aerosol Science", Academic Press, 1966, pp. 409.

[2] C. J. M. van Rijin, and M. C. Elwenpoek, "Micro Filtration Membrane Sieve with Silicon Micro Machining for Industrial and Biomedical Applications", Proceedings of IEEE the Eighth Workshop on Micro Electro Mechanical Systems, 1995, pp. 83-87.

[3] C. J. M. van Rijin, M. van der Wekken, W. Nijdam, and M. C. Elwenspoek, "Deflection and Maximum Load of Microfiltration Membrane Sieves Made with Silicon Micromachining", Journal of Microelectromechanical Systems, Vol. 6, No.1, March, 1997, pp. 48-54.

[4] G. Kittilsland, G. Stemme, and B. Norden, "A Submicron Paticle Filter in Silicon", Sensors and Actuators (A:Physical), Vol. 23, 1990, pp.904-907.

[5] M. Sekimoto, H. Yoshihara, and T. Ohkubo, "Silicon Nitride Single-Layer X-Ray Mask", Journal of Vacuum Science and Technology, Vol.21(4), November/December, 1982, pp.1017-1021.

[6] K. E. G. Wieghardt, "On the Resistance of Screens", The Aeronautical Quarterly, Vol. IV, February, 1953, pp.186-192.

[7] G. B. Schubauer, W. G. Spangenberg, P. S. Klebanoff, "Aerodynamic Characteristics of Damping Screens", N. A. C. A., Technical Notes 2001, 1950.

[8] T. Hasegawa, M. Suganuma, and H. Watanabe, "Anomaly of Excess Pressure Drops of the Flow Through Very Small Orifices", Physics of Fluids, Vol. 9, No.1, January 1997, pp. 1-3. 